\title{
Tuberculose mamária em paciente renal crônico: estudo de caso
}

\author{
Breast tuberculosis in a chronic kidney patient: case study
}

Tuberculosis de mama en una paciente renal crónica: estudio de caso

Rafael Marques de Figueiredo ${ }^{1 *}$, Anne Caroline da Silva Menezes ${ }^{1}$, Andrio Portilho de Souza ${ }^{1}$, Mariela Figueiredo Conceição².

\section{RESUMO}

Objetivo: Relatar um caso clínico de uma paciente renal crônica com tuberculose mamária. Detalhamento do caso: A paciente é portadora de doença renal crônica em tratamento com hemodiálise, esta apresentou nodulação na mama direita, de consistência firme, endurecida e dolorosa à palpação superficial. $O$ diagnóstico foi concluído por meio da clínica sugestiva, cultura para bacilo de Koch e histopatológico. O tratamento realizado foi o padrão para tuberculose extra-pulmonar com Rifampicina, Isoniazida, Pirazinamida e Etambutol por dois meses e continuidade com Rifampicina e Isoniazida por mais quatro meses. A paciente evoluiu com melhora do quadro clínico e redução do nódulo. Considerações finais: A tuberculose mamária é uma variante rara da tuberculose. É equivalente a menos que $0,1 \%$ das lesões deste órgão. A paciente foi submetida à terapia tuberculostática convencional e mostrou boa resposta. Este relato contribuiu para caracterizar a tuberculose mamária e para correlacionar a incidência de tuberculose em pacientes renais. Assim, atesta-se uma urgência em realizar pesquisas sobre a relação entre Insuficiência Renal Crônica e tuberculose.

Palavras-chaves: Tuberculose, Mama, Insuficiência renal, Manaus.

\begin{abstract}
Objective: To report a clinical case of a chronic renal patient with mammary tuberculosis. Case details: The patient has chronic kidney disease undergoing hemodialysis, who presented nodulation in the right breast, with a firm, hardened and painful consistency on superficial palpation. The diagnosis was made through the suggestive clinic, culture for Koch's bacillus and histopathology. The treatment performed was the standard for extra-pulmonary tuberculosis with Rifampicin, Isoniazid, Pyrazinamide and Etambutol for two months and continuity with Rifampicin and Isoniazid for another four months. The patient evolved with clinical improvement and reduction of the nodule. Final considerations: Breast tuberculosis is a rare variant of tuberculosis. It is equivalent to less than $0.1 \%$ of the injuries of this organ. The patient underwent conventional tuberculostatic therapy and showed a good response. This report contributed to characterize breast tuberculosis and to correlate the incidence of tuberculosis in renal patients. Thus, there is an urgent need to conduct research on the relationship between chronic renal failure and tuberculosis.
\end{abstract}

Keywords: Tuberculosis, Breast, Renal insufficiency, Manaus.

\section{RESUMEN}

Objetivo: Informar un caso clínico de una paciente renal crónica con tuberculosis mamaria. Detalle del caso: Paciente con enfermedad renal crónica sometida a hemodiálisis, presenta nódulos en mama derecha,

\footnotetext{
${ }^{1}$ Hospital Universitário Getúlio Vargas, Universidade Federal do Amazonas (HUGV/UFAM), Manaus - AM.

*E-mail: marquesrafael_@hotmail.com

${ }^{2}$ Clínica Renal de Manaus, Manaus - AM.
} 
de consistencia firme, endurecida y dolorosa a la palpación superficial. El diagnóstico se realizó mediante clínica sugerente, cultivo para bacilo de Koch e histopatología. El tratamiento realizado fue el estándar para la tuberculosis extrapulmonar con rifampicina, isoniazida, pirazinamida y etambutol durante dos meses y continuidad con rifampicina e isoniazida durante otros cuatro meses. El paciente evolucionó con mejoría del cuadro clínico y reducción del nódulo. Consideraciones finales: La tuberculosis de mama es una variante poco común de la tuberculosis. Equivale a menos del $0,1 \%$ de las lesiones de este órgano. El paciente se sometió a terapia tuberculostática convencional y mostró buena respuesta. Este informe contribuyó a caracterizar la tuberculosis de mama y a correlacionar la incidencia de tuberculosis en pacientes renales. Por lo tanto, existe una necesidad urgente de realizar investigaciones sobre la relación entre la insuficiencia renal crónica y la tuberculosis.

Palabras clave: Tuberculosis, Mama, Insuficiencia renal, Manaus.

\section{INTRODUÇÃO}

A tuberculose (TB) é uma doença estigmatizante e altamente contagiosa, transmitida pelo Mycobacterium tuberculosis por meio de gotículas respiratórias. É mais prevalente em países em desenvolvimento, onde questões socioeconômicas e sistemas de saúde pública ineficazes são incapazes de controlar a propagação da doença, apesar da disponibilidade de tratamentos eficazes (DAHER EF, et al., 2013). A tuberculose afeta milhões de pessoas em todo o mundo. A doença é endêmica na América Latina, onde as taxas de incidência variam de 25 a 149 casos por 100.000 habitantes. Brasil é um dos 20 países com maior número de casos (ZUMLA A, et al., 2013).

A ligação entre tuberculose (TB) e Insuficiência Renal Crônica (IRC) foi relatada pela primeira vez em uma série de casos de 1974 envolvendo pacientes em diálise. Desde então, inúmeras publicações confirmaram esta ligação, com coortes baseadas em hospitais e registros regionais mostrando de forma consistente que a diálise está associada a um risco aumentado de TB (DOBLER CC, et al., 2011).

Sabe-se pacientes portadores de Insuficiência Renal Crônica (IRC) apresentam risco aumentado de TB devido a uma resposta imune mediada por células prejudicada, que é responsável pela morte de organismos intracelulares como M. tuberculosis (ROMANOWSKI K, et al., 2016). As manifestações clínicas da TB em pacientes em diálise são bastante inespecíficas, dificultando o diagnóstico oportuno e retardando o início do tratamento curativo (JEBALI, et al., 2017). Foi observado uma redução da capacidade fagocítica e bactericida, aumentando a predisposição de infecções, principalmente as causadas por microbactérias, vírus e fungos. Os pacientes com IRC submetidos à diálise crônica possuem risco 25 vezes maior de desenvolver tuberculose, quando comparados com a população em geral (LUNDIN AP, et al., 1979).

A tuberculose inclui um espectro clínico variável e pode afetar diversos órgãos. O envolvimento extrapulmonar ocorre em 10 a $42 \%$ dos casos, e as formas extrapulmonares mais comuns são a tuberculose pleural, linfonodal e renal, nesta ordem (ZUMLA A, et al., 2013). Segundo Cengiz K (1996), em pacientes com IRC, a tuberculose é mais frequentemente extrapulmonar e a infecção geralmente ocorre nos primeiros 12 meses de diálise, sendo mais comum em pacientes sob hemodiálise crônica (HACHICHA J e JARRAYA A, 1989).

O sistema de dados renais dos EUA (U.S Renal Data System- USRD) verificou que idade, raça asiática e americana, tabagismo, índice de massa corporal reduzido, baixos níveis de albumina sérica, doença cardíaca isquêmica e anemia são fatores de risco significativos para o desenvolvimento de tuberculose em pacientes em tratamento com hemodiálise (LUNDIN AP, et al., 1979).

O primeiro caso de tuberculose mamária (TM) foi registrado por Cooper $A$ (1829), que o descreveu como "inchaço escrofuloso do seio". É uma doença rara, com incidência inferior a 0,1\% de todas as lesões mamárias nos países ocidentais e 3-4\% nas regiões endêmicas da tuberculose. Geralmente afeta mulheres multíparas jovens em lactação (entre 20 a 50 anos), embora também possa ser relatada em homens pré- 
púberes ou em mulheres idosas (LUH SP, et al., 2008). Mais comumente, a doença se apresenta como um nódulo no quadrante central ou superior externo da mama, enquanto vários nódulos aparecem com menos freqüência (MAROULIS I, et al., 2008). O médico pode diagnosticar erroneamente tuberculose de mama com carcinoma de mama ou abscesso (MIRSAEIDI SM, et al., 2007).

Com duração de alguns meses a alguns anos, a tuberculose mamária geralmente se apresenta como um nódulo mamário solitário no quadrante externo superior ou central devido à extensão frequente do linfonodo axilar à mama. A apresentação de massas mamárias múltiplas ou bilaterais é incomum. $\mathrm{O}$ caroço é geralmente irregular, mal definido e duro, simulando carcinoma. Pode ser doloroso, móvel ou fixo à pele ou músculo subjacente e parede torácica e também pode apresentar ulceração da pele sobrejacente, abscesso mamário, retração do mamilo e edema mamário. O diagnóstico de tuberculose mamária é ainda mais difícil de fazer e menos provável de ser considerado em homens (STRAZZANTI A, et al., 2018).

O diagnóstico padrão ouro da TM é por cultura bacteriológica do tecido mamário ou pela coloração de Ziehl Neelsen (ZN). No entanto, na TM os bacilos são isolados em apenas $25 \%$ dos casos, e os bacilos álcool-ácido resistentes (BAAR) são identificados em apenas $12 \%$ dos pacientes. Portanto, a demonstração de granulomas caseosos do tecido mamário e linfonodos envolvidos pode ser suficiente para o diagnóstico. A aspiração por agulha fina é o método invasivo inicial mais amplamente usado para o diagnóstico de tuberculose mamária (BAHAROON S, 2008).

A tuberculose mamária é classificada em 5 variedades patológicas. A forma nodular é a variedade mais comum e geralmente se apresenta como uma massa localizada de crescimento lento que progride para envolver a pele, pode ulcerar e formar seios da face. Histologicamente, esta forma é caracterizada por extensa caseação e pouca fibrose (MCKEOWN KC e WILKINSON KW, 1952).

O envolvimento tuberculoso da mama ocorre tanto pela inoculação direta dos bacilos por meio de escoriações no mamilo, o que é raro; ou mais comumente via disseminação linfática, hematogênica ou contígua. A via linfática é a via mais provável de envolvimento da mama que ocorre por extensão retrógrada do linfonodo axilar. Esta hipótese é apoiada pelo envolvimento de nódulos axilares, frequentemente nódulos ipsilaterais, em $50 \%$ a $75 \%$ dos casos de mastite tuberculosa (BAHAROON S, 2008).

Será relatado o caso de uma paciente com insuficiência renal crônica que apresentou nodulações na mama direita, tendo o diagnóstico de tuberculose mamária.

\section{DETALHAMENTO DO CASO}

Este artigo foi desenvolvido com a devida autorização, para isso foi elaborado o Termo de Consentimento Livre e Esclarecido, o qual foi assinado pela paciente. Paciente de 33 anos, sexo feminino, parda, natural de Santarém/PA iniciou quadro de edema em membros inferiores associado a lombalgias e vômitos. Viajou para a cidade de Manaus/AM para a realização de USG de rins onde foi verificado atrofia renal bilateral. Logo, recebeu indicação de hemodiálise três vezes por semana, a qual foi realizada em uma clínica especializada em tratamento renal.

Em meados de 2016, iniciou episódios de palpitações esporádicas, febre e calafrios que pioravam durante a hemodiálise, sem horário definido e que melhoravam após uso de sintomáticos. Em agosto de 2016, evoluiu com quadro ascítico, edema em membros inferiores, calafrios, astenia, plenitude pós-prandial, lombalgia, parestesias em membro superior esquerdo e hemitórax esquerdo com irradiação para o dorso e região auricular esquerda. Realizou hemoculturas ( 2 amostras) negativas durante vigência de quadro febril. Foi realizada pesquisa de fungos em liquídoascítico, sendo evidenciada ao exame direto presença de leveduras e hifas hialinas largas e sem septos. Paciente realizou tratamento com Anfotericina B por 20 dias.

Em dezembro de 2016, a paciente evoluiu com nodulações em mama direita. Ao exame físico das mamas: nódulo em quadrante medial inferior de mama direita com cerca de $4 \mathrm{~cm}$ de comprimento, delimitação irregular, doloroso a palpação superficial, consistência firme, espessamento cutâneo e sem linfoadenomegalia axilar à palpação. 
Ao exame mamográfico, USG de mama (30/12/2016) evidenciou nódulos sólidos, com margens circunscritas, nítida transição entre os tecidos adjacentes, forma ovalada, com maior eixo paralelo a pele, conforme seta verde indicada na Figura 1. Com textura hipoecóicas com paredes levemente espessadas e coleção espessa com "debris" grossos e sinais de edema em tecidos adjacentes, assim distribuídas: - QII, às $04 \mathrm{hs}$, medindo: $1,5 \times 1,1 \times 1,1 \mathrm{~cm}$ e às $5 \mathrm{hs}$, medindo respectivamente $2,3 \times 1,1 \times 1,3 \mathrm{~cm}$ identificado pela seta azul na Figura 2 e 1,2×1,0×1,1 cm apontado pela seta amarela na Figura 3. BIRADS- categoria 5.

Figura 1 - USG mamária mostrando imagens nodulares. Predominantemente hipoecóicas com paredes levemente espessadas e sinais de edema em tecidos adjacentes.

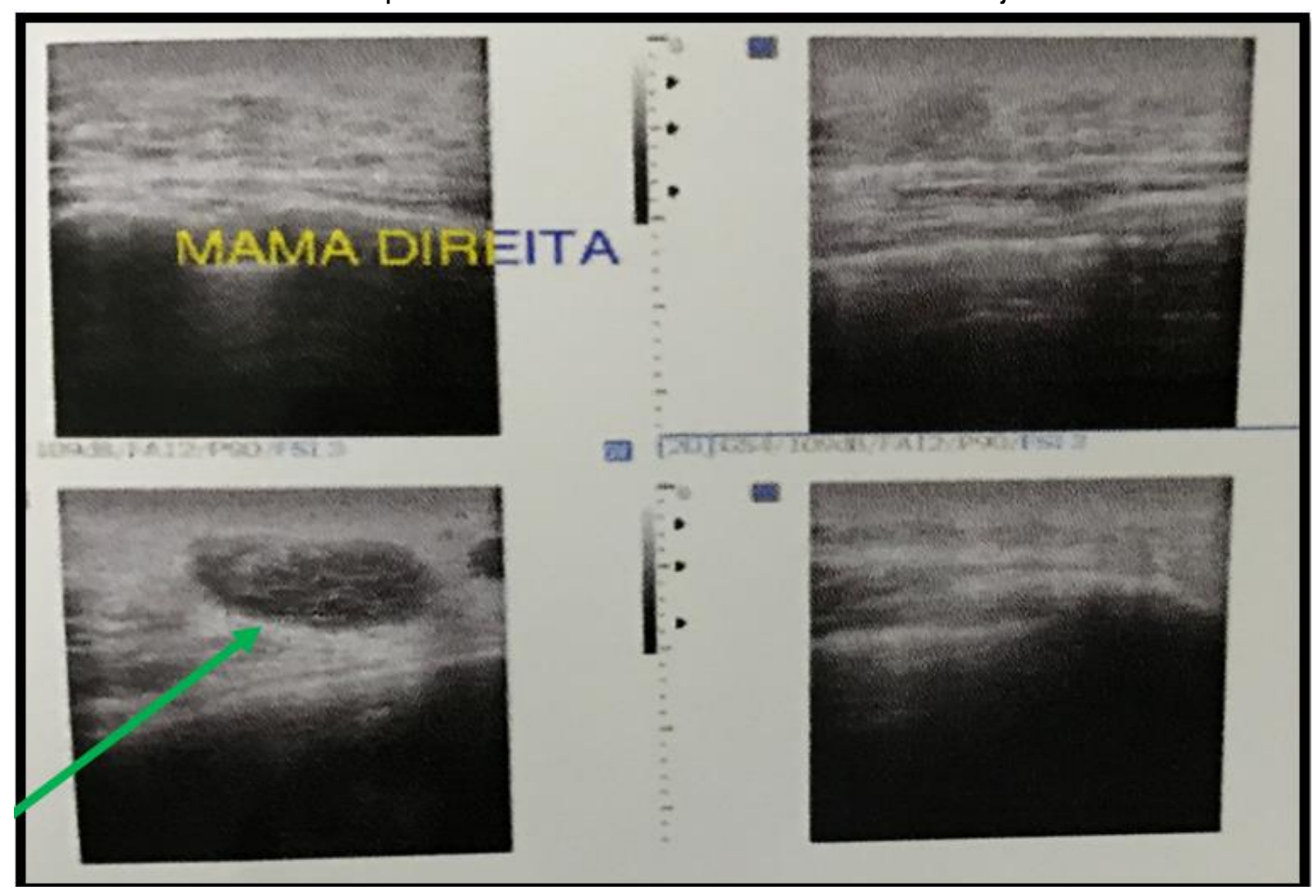

Fonte: Figueiredo RM, et al., 2021.

Figura 2 - USG mamária mostrando nódulo hipoecóico, medindo: 2,3×1,1×1,3cm.

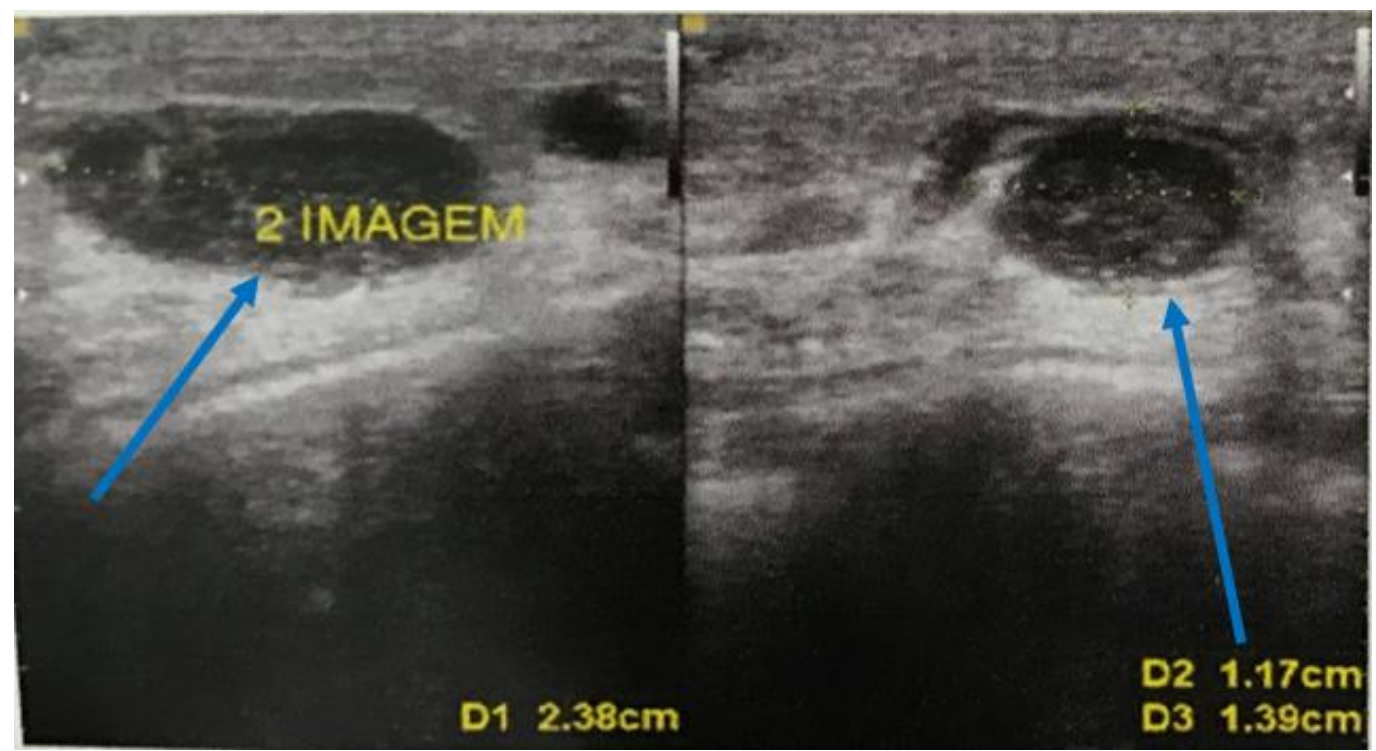

Fonte: Figueiredo RM, et al., 2021. 
Figura 3 - USG mamária mostrando nódulo medindo:1,2×1,0x1,19cm.

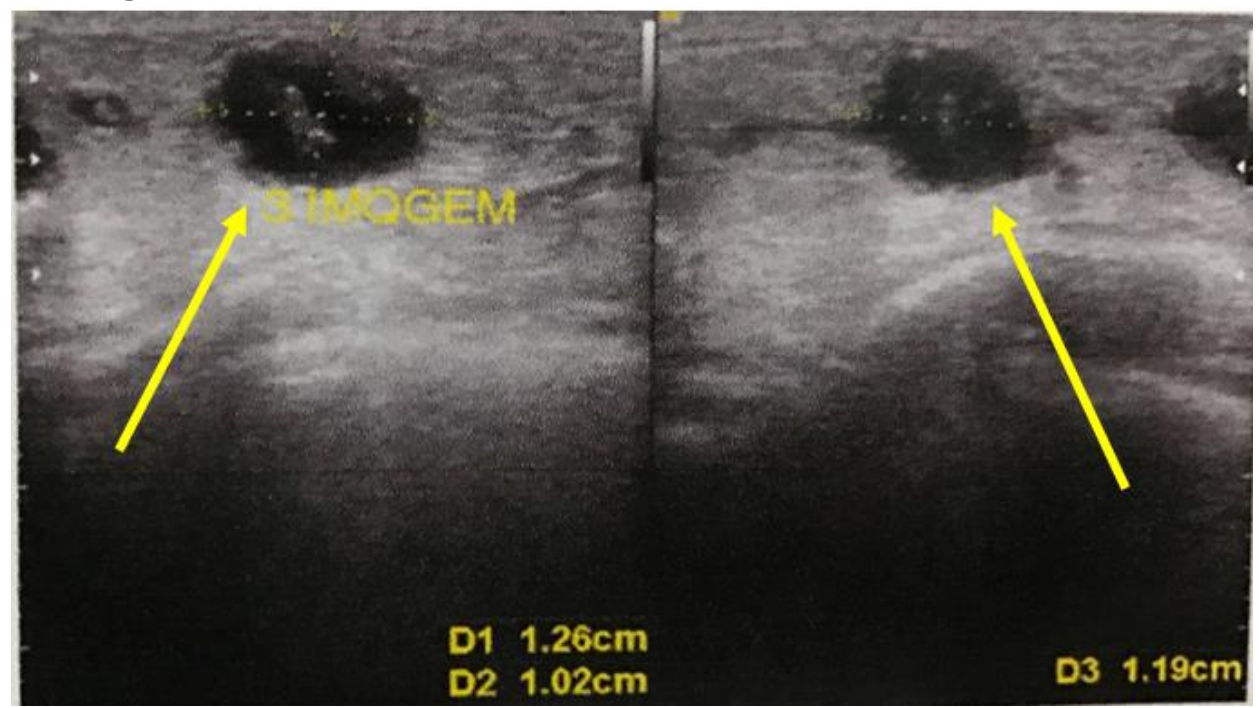

Fonte: Figueiredo RM, et al., 2021.

Apresentava achados altamente sugestivos de malignidade, no entanto durante realização de biópsia de mama notou-se presença de secreção purulenta. O exame histopatológico apresentou-se como granuloma com necrose de caseificação e denso infiltrado inflamatório linfoistiocitário.

Foi realizada pesquisa de BAAR em amostra de aspirado de mama(04/01/2017) com resultado positivo. A paciente apresentava os seguintes resultados sorológicos: anti- HIV- não reagente; HBsAg- não reagente; Anti-HBs- reagente; HBeAg- não reagente; anti- HCV- não reagente; CardiolipinalgM- não reagente; Ceruloplasmina- não reagente. Pelos aspectos clínicos, radiográficos e microbiológicos iniciou-se o esquema para tratamento de tuberculose extra-pulmonar com Rifampicina, Isoniazida, Pirazinamida e Etambutol por dois meses e continuidade com Rifampicina e Isoniazida por mais quatro meses. Confirmando o diagnóstico o tratamento adequado, a paciente evoluiu com regressão do nódulo e melhora do quadro geral.

\section{DISCUSSÃO}

As infecções continuam sendo uma causa significativa de morbidade e mortalidade em pacientes em diálise. A tuberculose da mama é uma doença rara, principalmente porque órgãos ou tecidos como a mama, músculo esquelético e baço são mais resistentes à infecção, dificultando a sobrevivência e a multiplicação dos bacilos da tuberculose (DA SILVA BB, et al., 2009). A mastite tuberculosa costuma ser unilateral, raramente infecta pacientes do sexo masculino e deve ser considerada em estados de imunodeficiência como no caso relatado, cujo paciente possuía Insuficiência Renal Crônica (MIRSAEIDI SM, et al., 2007).

A tuberculose mamária representa uma doença rara que deve ser sempre suspeitada na avaliação de casos de abscessos, fístulas ou nódulos mamários, com má resposta ao tratamento antibiótico clássico não tuberculoso. Os médicos devem considerar essa entidade clínica, muitas vezes imitando o câncer de mama, especialmente em tempos de picos migratórios (MARINOPOULOS S, et al., 2012).

A apresentação clínica mais comum da mastite tuberculosa é a de um nódulo duro unilateral, solitário, mal definido, situado no quadrante externo superior ou central. A lesão pode ser indistinguível do carcinoma de mama, sendo irregular, dura e, às vezes, fixada na pele ou músculo ou mesmo na parede torácica. Nódulos múltiplos e envolvimento bilateral são incomuns e ocorrem em menos de $3 \%$ das pacientes. A lesão pode progredir para úlcera tuberculosa sobre a pele da mama e abscesso mamário tuberculoso com ou sem secreção dos seios da face (BAHAROON S, 2008). Concordando com a literatura a paciente possuía a forma mais predominante uma tuberculose mamária na forma nodular, unilateral, indolor no início, sem envolvimento axilar e sem envolvimento fistular. 
Não há diretrizes específicas disponíveis para a terapia da tuberculose mamária, esta geralmente segue as diretrizes usadas para a tuberculose pulmonar. A taxa de sucesso da terapia médica se aproxima de 95\% na maioria dos esquemas de seis meses de terapia antituberculose (2 meses de isoniazida, rifampicina, pirazinamida e etambutol / 4 meses de isoniazida e rifampicina (BAHAROON S, 2008). A paciente respondeu adequadamente ao esquema convencional, não havendo falha terapêutica ou necessidade de procedimentos cirúrgicos adjuvantes. As intervenções cirúrgicas são necessárias em até $14 \%$ dos pacientes, devido à falha da quimioterapia ou à grande extensão da lesão (TEWARI e MALLIKA HS, 2005).

O tratamento da TB em diálise é difícil e desafiador. Há uma alta incidência de efeitos colaterais dos medicamentos anti-TB, principalmente neuropsiquiátricos, hepáticos e gastrointestinais. Em conclusão, TB em pacientes em diálise carrega alta morbidade e mortalidade (VIKRANT S, 2019).

Relatos na literatura demonstram que há um aumento da frequência de TB extrapulmonar, a apresentação clínica é atípica e os sintomas e achados são inespecíficos. Esta apresentação atípica pode muitas vezes levar a um atraso no diagnóstico preciso e na terapia, às vezes resultando na morte do paciente. Portanto, os médicos devem sempre ter um alto grau de suspeita e considerar a possibilidade de TB sempre que confrontados com pacientes em diálise que apresentem sintomas gerais como febre, perda de peso e linfadenopatia. O diagnóstico exigiria então o isolamento dos bacilos álcool-ácido resistentes, o achado de um granuloma caseoso típico na biópsia ou a recuperação dos bacilos da tuberculose a partir da cultura do material de biópsia (VIKRANT S, 2019).

A tuberculose mamária, apesar de uma afecção rara, é um diagnóstico que precisa ser suspeitado, principalmente em pacientes que apresentem lesão mamária de evolução subaguda ou crônica com má resposta a terapia antimicrobiana convencional. O diagnóstico baseia-se na clínica e em achados histopatológicos, sendo o diagnóstico etiológico muito difícil. A terapia tuberculostática convencional apresenta ótimos resultados, devendo ser sempre considerada como primeira escolha mesmo nos indivíduos portadores de insuficiência renal crônica.

Há uma necessidade de orientação clara e consistente, apropriada para epidemiologia regional, para facilitar protocolos adequados para triagem, diagnóstico e tratamento de TB em pacientes com IRC. Diretrizes baseadas em evidências e rigorosamente desenvolvidas reduzem a variabilidade do atendimento, melhoram os resultados dos pacientes e atualizam as deficiências na prestação de cuidados de saúde em pacientes com IRC. Sem essas diretrizes, a TB continuará a afligir as populações com IRC e provavelmente se tornará um fator cada vez mais importante para a disseminação da TB em populações vulneráveis (LEVEY AS, et al., 2005).

Assim, acredita-se que haja uma urgência em realizar pesquisas sobre a relação entre Insuficiência Renal Crônica e tuberculose, mais especificamente tuberculose mamária, relação esta que não está muito bem descrita na literatura. Novas pesquisas para orientar as políticas são necessárias, incluindo estudos robustos da associação de ambas as condições, o efeito do estágio de IRC no desempenho das modalidades de triagem de TB, estudos randomizados, controlados e bem planejados que examinem regimes de tratamento preventivo. Sem esses avanços, as estratégias de prevenção, detecção e tratamento da tuberculose relacionada ao IRC não podem ser melhor definidas.

\section{REFERÊNCIAS}

1. BAHAROON S. Tuberculosis of the breast. Ann Thorac Med. 2008 Jul;3(3):110-4.

2. CENGIZ K. Increase incdence of tuberculosis in patients undergoing hemodialysis. Nephron 1996; 73: 421-4

3. COOPER A. Illustrations of the diseases of the breast. London: Longman, Rees, Orme, Brown and Green; 1829.

4. DAHER EF, et al. Renal tuberculosis in the modern era. Am J Trop Med Hyg 2013; 88: 54-64.

5. DA SILVA BB, et al. Tuberculosis of the breast: analysis of 20 cases and a literature review. Transactions of the Royal Society of Tropical Medicine and Hygiene 2009; 103: 559-63.

6. DOBLER CC, et al. Risk of tuberculosis in dialysis patients: a nationwide cohort study. PloS One. $2011 ; 6$ : e29563.

7. HACHICHA J, JARRAYA A. High incidence of tuberculosis in chronic dialysis patients in developing countries. Nephron1989; 52: 189. 
8. JEBALI H, et al. The diagnosis of tuberculosis in dialysis patients. Saudi J Kidney Dis Transpl. 2017; 28: 1362-1368.

9. LEVEY AS, et al. Definition and classification of chronic kidney disease: a position statement from Kidney Disease: Improving Global Outcomes (KDIGO). Kidney Int. 2005; 67: 2089-2398.

10. LUH SP, et al. Surgical treatment for primary mammary tuberculosis - report of three octogenarian cases and review of literature. Breast Journal 2008; 14: 311-2

11. LUNDIN AP, et al. Tuberculosis in patients undergoing maintenancehemodialysis. Amer J Med 1979; 67: $597-602$.

12. MARINOPOULOS S, et al. Breast tuberculosis: Diagnosis, management and treatment. Int J Surg Case Rep. 2012; 3(11): 548-5018.

13. MAROULIS I, et al. Mammary tuberculosis mimicking breast cancer: a case report. Journal of Medicine Case Reports 2008; $2: 34$.

14. MCKEOWN KC, WILKINSON KW. Tuberculosis of the breast. Br J Surg. 1952;39:420-9.

15. MIRSAEIDI SM, et al. Tuberculosis of the breast: report of 4 clinical cases and literature review. East Mediterranean Health Journal 2007; 13: 670-6.

16. ROMANOWASKI K, et al. Tuberculosis and chronic kidney disease: An emerging global syndemic. Kidney Int. 2016;90: 34-40.

17. STRAZZANTI, C, et al. Breasttuberculosis cases rising in Sicilylnt J Surg Case Rep, 53 (January (1)) (2018), pp. 9-12

18. TEWARI, MALLIKA, HS. Breast tuberculosis: diagnosis, clinical features \& management - Indian Journal of Medical Research, 2005.

19. VIKRANT S. Tuberculosis in dialysis: Clinical spectrum and outcome from an endemic region. Hemodial Int. 2019 Jan;23(1):88-92. doi: 10.1111/hdi.12693. Epub 2018 Oct 5. PMID: 30289617.

20. ZUMLA A, et al. Tuberculosis. N Engl J Med 2013; 368:745-755. 